\title{
Slow Relaxation Mode in Mixtures of Water and Organic Molecules: Supramolecular Structures or Nanobubbles?
}

\author{
Fan Jin, ${ }^{\dagger}$ Jing Ye, ${ }^{\dagger}$ Liangzhi Hong, ${ }^{\dagger}$ Hiufung Lam, ${ }^{\dagger}$ and Chi $\mathrm{Wu}^{*, \dagger, \dagger}$ \\ Department of Chemistry, The Chinese University of Hong Kong, Shatin, N.T., Hong Kong, China, and \\ The Hefei National Laboratory of Physical Science at Microscale, Department of Chemical Physics, \\ The University of Science and Technology of China, Hefei, Anhui 230026, China
}

Received: December 17, 2006; In Final Form: January 7, 2007

\begin{abstract}
Aqueous solutions of tetrahydrofuran, ethanol, urea, and $\alpha$-cyclodextrin were studied by a combination of static and dynamic laser light scattering (LLS). In textbooks, these small organic molecules are soluble in water so that there should be no observable large structures or density fluctuation in either static or dynamic LLS. However, a slow mode has been consistently observed in these aqueous solutions in dynamic LLS. Such a slow mode was previously attributed to some large complexes or supramolecular structures formed between water and these small organic molecules. Our current study reveals that it is actually due to the existence of small bubbles $(\sim 100 \mathrm{~nm}$ in diameter) formed inside these solutions. Our direct evidence comes from the fact that it can be removed by repeated filtration and regenerated by air injection. Our results also indicate that the formation of such nanobubbles in small organic molecule aqueous solutions is a universal phenomenon. Such formed nanobubbles are rather stable. The measurement of isothermal compressibility confirms the existence of a low density microphase, presumably nanobubbles, in these aqueous solutions. Using a proposed structural model, that is, each bubble is stabilized by small organic molecules adsorbed at the gas/water interface, we have, for the first time, estimated the pressure inside these nanobubbles.
\end{abstract}

\section{Introduction}

It is fair to say that liquid water is the most complicated fluid in nature even it has a very simple chemical structure. Many physical and chemical properties of liquid water are abnormal and perplexing. ${ }^{1-3}$ Numerous theoretical, ${ }^{4-6}$ experimental, ${ }^{7-10}$ and computer simulation ${ }^{11-15}$ efforts have been devoted to construct water structures and explain these abnormal phenomena. Nowadays, liquid water structures grasped by a "two-state model" are not surprising anymore. ${ }^{16,17}$ Namely, liquid water exists in an "open" state and a "denser" state, ${ }^{18}$ essentially determined by a "nonlocal potential", rooting in a competition between Van der Waals interaction and hydrogen bonding. ${ }^{19}$ These two states of liquid water were well reviewed. ${ }^{20-22}$

Note that aqueous solutions are more important than pure liquid water because they are more closely related to various applications, particularly in biological systems. Hydrophobic interaction $^{23-25}$ and association ${ }^{26-29}$ are two core problems in aqueous solutions. They play a central role in self-assembly or organization of solute molecules in aqueous solutions, such as the protein folding and the formation of surfactant micelles, membranes, and various mesophases. ${ }^{30-34}$ Pratt et al. ${ }^{35-38}$ made some breakthrough in this direction by using an information theory, ${ }^{39,40}$ which enables us to understand hydrophobic interaction on a molecular scale. However, many unsolved problems and challenges still exist in this active research area, such as hydrophobic interaction on mesoscopic length scales, ${ }^{41,42}$ multibody interaction, ${ }^{43-45}$ wetting and dewetting, ${ }^{46-48}$ and longrange hydrophobic interaction. ${ }^{49,50}$

* To whom correspondence should be addressed. The Hong Kong address should be used for all correspondence.

$\dagger$ The Chinese University of Hong Kong.

$\div$ The University of Science and Technology of China.
The aqueous solutions such as tetrahydrofuran (THF)/water, urea/water, ethanol/water, and sugar/water have been extensively investigated before. ${ }^{51-61}$ In dynamic laser light scattering (LLS) measurements of THF aqueous solution, we encountered a "slow mode" (corresponding to a length scale of $\sim 100 \mathrm{~nm}$ ) $\sim 10$ years ago without any reasonable and rational explanation. In this study, we found that such a slow mode exists in many other aqueous solutions, such as urea/water, ethanol/water, sugar/ water, and surfactant/water. These similar experimental phenomena strongly imply that the slow mode is universal in aqueous solutions of small organic molecules. Other research groups also noted such a phenomenon ${ }^{62-64}$ and attributed it to hydrophobic association of solute molecules and water, namely, loosely structured supramolecules or complexes assembled by small organic solutes and many water molecules. At first, we also tried to explain the slow mode in a similar fashion.

However, more and more of our own experimental data and other literature reports have gradually led us to question such an explanation. The reasons are as follows. First, the slow mode has only been observed in dynamic LLS experiments but not by other experimental methods even if they have a similar mesoscopic observation length scale. Second, recent theories and computer simulations have not predicted such a long-range correlation or large supramolecular structure. Third, we found that its related dynamic correlation length is independent of the concentration of small organic solutes. Fourth, it cannot be removed by a simple filtration, indicating that these so-called supramolecules would have an open structure even if they did exist.

On the other hand, we noted that "long-range $(\sim 100 \mathrm{~nm})$ hydrophobic interaction" was observed between two macroscopic hydrophobic surfaces, ${ }^{65-67}$ which could not be satisfactorily explained by any theory or computer simulations. ${ }^{68,69} \mathrm{In}$ 
principle, hydrophobic attraction cannot extend to such a length scale between two macroscopic hydrophobic surfaces. Tyrell and Attard ${ }^{70}$ found that some small bubbles could spontaneously form at a macroscopic hydrophobic surface, which shed light on such long-range hydrophobic interaction and led to some reasonable explanation. ${ }^{71,72}$ Previously, Bilgram et al. ${ }^{73}$ also observed a new slow relaxation mode in dynamic light scattering measurements of water near the ice/water interface during directional solidification. Several different explanations for this mode were proposed, but eventually, Williams et al. ${ }^{74}$ showed that it is due to small bubbles of gas originally dissolved in the water that is rejected during solidification.

It was these works that inspired us to look at the observed slow mode in dynamic LLS from a different angle, that is, whether the mesoscopic or microscopic hydrophobic surface can also lead to the formation of nanobubbles. It should be noted that, in general, nanobubbles are less understood and some of their related problems are complicated, such as how they affect the inhomogeneity of a fluid in the mesoscopic scale, ${ }^{75}$ how they are stabilized, ${ }^{76}$ what mesoscopic hydrophobic interaction is, ${ }^{77}$ and how high the pressure is inside them. ${ }^{78}$

\section{Experimental Section}

Materials and Solution Preparation. $\alpha$-Cyclodextrin $(\alpha-$ $\mathrm{CD},>99.9 \%$ ), urea $(>99 \%)$, tetrahydrofuran (THF, $>99.8 \%$ ), and ethanol $(>99.9 \%)$ were, respectively, purchased from Aldrich, Scharlau, LAB-SCAN, and Merck KGaA. THF and urea were, respectively, purified by distillation and recrystallization. ${ }^{79} \alpha-C D$ and ethanol were used without further purification. Water was purified with an inverse osmosis filtration (Nano Pure, Barnstead) and filtrated with a Millipore PTFE $0.45 \mu \mathrm{m}$ hydrophilic filter. Its resistivity was $18.2 \mathrm{M} \Omega \cdot \mathrm{cm}$ at $20^{\circ} \mathrm{C}$. The stirring-mixed aqueous solutions were prepared at $\sim 20^{\circ} \mathrm{C}$ and stood for $\sim 12 \mathrm{~h}$ to ensure a complete dissolution. For laser light scattering (LLS) experiments, dust particles were removed from these aqueous solutions by one-time filtration using a normal syringe and a Millipore $0.45 \mu \mathrm{m}$ PTFE hydrophilic filter. Each solution was also repeatedly filtrated by using a tubing flex pump (Master Flex) and different filters, including Millipore $0.45 \mu \mathrm{m}$ PTFE and Nylon hydrophilic filters and a Whatman $0.02 \mu \mathrm{m}$ inorganic filter. In the repeated filtration, each solution was circulated to pass through the filter and the LLS cuvette in a closed loop.

Laser Light Scattering. A commercial LLS instrument (ALV5000) with a vertically polarized $22 \mathrm{~mW} \mathrm{He}-\mathrm{Ne}$ laser head (632.8 nm, Uniphase) was used. The angular range was $6-155^{\circ}$. In LLS, the essential measurement length scale is the reciprocal of the scattering vector $(\mathbf{q}),{ }^{80}$ that is, $1 / q$, with

$$
q \equiv|\mathbf{q}|=\frac{4 \pi n}{\lambda_{0}} \sin \frac{\theta}{2}
$$

where $n$ is the refractive index of the scattering medium, $\lambda_{0}$ is the wavelength of the incident beam in vacuum, and $\theta$ is the scattering angle. For a given $\theta$, the scattering intensity was measured by a photon-counting system composed of a high quantum efficiency avalanche photodiode detector (ALV High Q.E. APD) and a single-photon-counting module. The scattering intensity was converted to a photon correlation function $\left(G^{(2)}\right.$ $(\tau, q) \equiv\langle n(0, q) n(\tau, q)\rangle)$ with the delay time $(\tau)$ in a broad range $\left(10^{-7}-10^{3} \mathrm{~s}\right)$, which was measured by an ALV multiple-tau digital time correlator. In Poisson statistics, ${ }^{81} G^{(2)}(\tau, q)$ and the average photon counts per time $(\langle n(q)\rangle)$ are directly related to the normalized intensity-intensity time correlation function $\left(g^{(2)}\right.$ - $\left.(\tau, q) \equiv\langle I(0, q) I(\tau, q)\rangle /\langle I(q)\rangle^{2}\right)$ and the time-average intensity $(\langle I(q)\rangle)$. The measured relative errors of $g^{(2)}(\tau, q)$ and $\langle I(q)\rangle$ in this study were controlled to be less than 0.5 and $1 \%$, respectively. The scattering cell was thermostated at $25.00 \pm$ $0.05{ }^{\circ} \mathrm{C}$.

In static LLS, the scattering intensity $\langle I(q)\rangle$ is related to the Fourier transform of a density fluctuation correlation function $\left(\left\langle\Delta \rho\left(\mathbf{r}_{1}\right) \Delta \rho\left(\mathbf{r}_{2}\right)\right\rangle\right)$ as ${ }^{82}$

$$
\langle I(q)\rangle \propto \iint \mathrm{d} \mathbf{r}_{1} \mathrm{~d} \mathbf{r}_{2}\left\langle\Delta \rho\left(\mathbf{r}_{1}\right) \Delta \rho\left(\mathbf{r}_{2}\right)\right\rangle \mathrm{e}^{-i \mathbf{q} \cdot\left(\mathbf{r}_{1}-\mathbf{r}_{2}\right)}
$$

Generally speaking, the density correlation function $\left(\left\langle\Delta \rho\left(\mathbf{r}_{1}\right) \Delta \rho\right.\right.$ $\left.\left.\left(\mathbf{r}_{2}\right)\right\rangle\right)$ is a function of the distance $\left(r=\left|\mathbf{r}_{1}-\mathbf{r}_{2}\right|\right)$ between $\mathbf{r}_{1}$ and $\mathbf{r}_{2}$ in a homogeneous system with an Ornstein-Zernike form $^{83}$

$$
\langle\Delta \rho(0) \Delta \rho(r)\rangle \propto \frac{1}{r} \exp \left(-\frac{r}{\xi_{\mathrm{S}}}\right)
$$

where $\zeta_{\mathrm{S}}$ is the static correlation length. A combination of eqs 2 and 3 results in

$$
\langle I(q)\rangle \propto \frac{\zeta_{\mathrm{S}}^{2}}{1+q^{2} \xi_{\mathrm{S}}^{2}}
$$

Therefore, the plot of $1 /\langle I(q)\rangle$ versus $q^{2}$ can lead to $\zeta_{\mathrm{s}}$. Note that the resolution of static LLS is experimentally limited by the maximum scattering angle. In other words, $\langle I(q)\rangle$ becomes independent of $q$ when $\xi_{S}$ is shorter than $\sim 10 \mathrm{~nm}$. Equation 2 shows that the scattering intensity at zero angle is proportional to the total density fluctuation $\left(\left\langle\Delta \rho^{2}\right\rangle_{V}\right)$ in the scattering volume (V) as $^{84}$

$$
\langle I(0)\rangle \propto \iint \mathrm{d} \mathbf{r}_{1} \mathrm{~d} \mathbf{r}_{\mathbf{2}}\left\langle\Delta \rho\left(\mathbf{r}_{1}\right) \Delta \rho\left(\mathbf{r}_{2}\right)\right\rangle=\left\langle\Delta \rho^{2}\right\rangle_{V}
$$

For a two-component grand canonical ensemble (general solution system), $\left\langle\Delta \rho^{2}\right\rangle_{V}$ is the summation of the solvent molecule density fluctuation $\left(\left\langle\Delta \rho_{\mathrm{s}}{ }^{2}\right\rangle_{V}\right)$ and the concentration fluctuation of solute molecules $\left(\left\langle\Delta c^{2}\right\rangle_{V}\right)$ in the scattering volume, that is, ${ }^{83}$

$$
\left\langle\Delta \rho^{2}\right\rangle_{V}=\left\langle\Delta \rho_{\mathrm{s}}^{2}\right\rangle_{V}+\left\langle\Delta C^{2}\right\rangle_{V}
$$

Further, $\left\langle\Delta \rho_{\mathrm{s}}{ }^{2}\right\rangle_{V}$ and $\left\langle\Delta C^{2}\right\rangle_{V}$ are, respectively, proportional to the isothermal compressibility $\left(\kappa_{\mathrm{T}}=-1 / V(\partial V / \partial P)_{N, T}\right)$ and the osmotic compressibility $(\Pi)^{84,85}$ as $\left\langle\Delta \rho_{\mathrm{s}}{ }^{2}\right\rangle_{V} \sim k_{\mathrm{B}} T \rho \kappa_{\mathrm{T}} / V$ and $\left\langle\Delta C^{2}\right\rangle_{V} \sim k_{\mathrm{B}} T C(\partial C / \partial \Pi)_{V, T} / V$. Therefore, we have

$$
\langle I(0)\rangle \propto \frac{k_{\mathrm{B}} T}{V}\left[\rho \kappa_{\mathrm{T}}+C\left(\frac{\partial C}{\partial \Pi}\right)_{V, T}\right]
$$

where $k_{\mathrm{B}}$ is the Boltzemann constant and $T$ is the absolute temperature. On the basis of eq $2, g^{(2)}(\tau, q)$ can be further written as $^{85}$

$$
\begin{array}{r}
\iiint \int \mathrm{d} \mathbf{r}_{1} \mathrm{~d} \mathbf{r}_{2} \mathrm{~d} \mathbf{r}_{3} \mathrm{~d} \mathbf{r}_{4}\left\langle\Delta \rho\left(\mathbf{r}_{1}, 0\right) \Delta \rho\left(\mathbf{r}_{2}, 0\right) \Delta \rho\left(\mathbf{r}_{3}, \tau\right)\right. \\
\left.\Delta \rho\left(\mathbf{r}_{4}, \tau\right)\right\rangle \mathrm{e}^{-i \mathbf{q} \cdot\left(\mathbf{r}_{1}-\mathbf{r}_{2}\right)-i \mathbf{q} \cdot\left(\mathbf{r}_{3}-\mathbf{r}_{4}\right)}
\end{array}
$$

Since $\langle I(q)\rangle=\left\langle E(q) E^{*}(q)\right\rangle$, the normalized field-field time correlation function $\left[g^{(1)}(\tau, q) \equiv\left\langle E(0, q) E^{*}(\tau, q)\right\rangle /\langle I(q)\rangle\right]$ can be written as ${ }^{86}$

$$
g^{(1)}(\tau, q) \propto \iint \mathrm{d} \mathbf{r}_{1} \mathrm{~d} \mathbf{r}_{2}\left\langle\Delta \rho\left(\mathbf{r}_{1}, 0\right) \Delta \rho\left(\mathbf{r}_{2}, \tau\right)\right\rangle \mathrm{e}^{-i \mathbf{q} \cdot\left(\mathrm{r}_{1}-\mathbf{r}_{2}\right)}
$$


Using the Wick theorem, we can factor $\left\langle\Delta \rho\left(\mathbf{r}_{1}, 0\right) \Delta \rho\left(\mathbf{r}_{2}, 0\right) \Delta \rho\right.$ $\left.\left(\mathbf{r}_{\mathbf{3}}, \tau\right) \Delta \rho\left(\mathbf{r}_{\mathbf{4}}, \tau\right)\right\rangle$ as $^{87}$

$$
\begin{aligned}
& \left\langle\Delta \rho\left(\mathbf{r}_{1}, 0\right) \Delta \rho\left(\mathbf{r}_{2}, 0\right) \Delta \rho\left(\mathbf{r}_{3}, \tau\right) \Delta \rho\left(\mathbf{r}_{4}, \tau\right)\right\rangle= \\
& \left\langle\Delta \rho\left(\mathbf{r}_{1}, 0\right) \Delta \rho\left(\mathbf{r}_{2}, 0\right)\right\rangle\left\langle\Delta \rho\left(\mathbf{r}_{3}, \tau\right) \Delta \rho\left(\mathbf{r}_{4}, \tau\right)\right\rangle+ \\
& \left\langle\Delta \rho\left(\mathbf{r}_{1}, 0\right) \Delta \rho\left(\mathbf{r}_{3}, \tau\right)\right\rangle\left\langle\Delta \rho\left(\mathbf{r}_{2}, 0\right) \Delta \rho\left(\mathbf{r}_{4}, \tau\right)\right\rangle+ \\
& \left\langle\Delta \rho\left(\mathbf{r}_{1}, 0\right) \Delta \rho\left(\mathbf{r}_{\mathbf{4}}, \tau\right)\right\rangle\left\langle\rho\left(\mathbf{r}_{2}, 0\right) \Delta \rho\left(\mathbf{r}_{3}, \tau\right)\right\rangle
\end{aligned}
$$

Generally, the second term only contributes to the scattering intensity at zero angle so that it is usually omitted. As a result, eq 8 can be rewritten as ${ }^{88}$

$$
g^{(2)}(\tau, q)=1+\left|g^{(1)}(\tau, q)\right|^{2} \quad \text { (the Siegert relation) }
$$

$\left\langle\Delta \rho\left(\mathbf{r}_{1}, 0\right) \Delta \rho\left(\mathbf{r}_{2}, \tau\right)\right\rangle$ can be expanded as a series of Gaussian functions, written in an integration form as follows:

$$
\left\langle\Delta \rho\left(\mathbf{r}_{1}, 0\right) \Delta \rho\left(\mathbf{r}_{2}, \tau\right)\right\rangle=\int \mathrm{d} D(4 \pi D \tau)^{-3 / 2} G(D) \mathrm{e}^{-\frac{\left(\mathbf{r}_{1}-\mathbf{r}_{2}\right)^{2}}{4 D \tau}}
$$

where $D$ is the variance of the Gaussian function and $G(D)$ is the expanded coefficient of the series in a discrete form or a distribution of $D$, characterizing dynamic properties of a system. $D$ can be related to the translational diffusion coefficient if the density fluctuation is completely induced by the random Brownian motion. A combination of eqs 9 and 12 leads to ${ }^{89}$

$$
\left|g^{(1)}(\tau, q)\right| \propto \int \mathrm{d} D G(D) \mathrm{e}^{-D q^{2} \tau}
$$

$G(D)$ can be obtained from the Laplace inversion of a measured $g^{(2)}(\tau, q)$ value by using a CONTIN program. With the StokesEinstein equation, it is convenient to introduce a dynamic correlation length $\left(\xi_{D}\right)$ defined as ${ }^{90}$

$$
\xi_{\mathrm{D}}=\frac{k_{\mathrm{B}} T}{3 \pi \eta D}
$$

where $\eta$ is the solvent viscosity. In comparison with $\xi_{\mathrm{s}}$, dynamic LLS leads to a much broader range of $\zeta_{\mathrm{D}}\left(10^{-1}-10^{3} \mathrm{~nm}\right)$. In this study, both fast and slow relaxation modes were observed so that we can write $g^{(1)}(\tau, q)$ as ${ }^{80}$

$$
\left|g^{(1)}(\tau, q)\right|=A_{\text {fast }}(q) \mathrm{e}^{-\left\langle D_{\text {fast }}\right\rangle q^{2} \tau}+\left[1-A_{\text {fast }}(q)\right] \mathrm{e}^{-\left\langle D_{\text {slow }}\right\rangle q^{2} \tau}
$$

where $\mathrm{A}_{\text {fast }}(q)$ is the scattering intensity contribution of the fast mode. The time-average scattering intensity of each mode can be calculated from $\langle I(q)\rangle$ measured in static LLS, that is, $\left\langle I_{\mathrm{fast}^{-}}\right.$ $(q)\rangle=\langle I(q)\rangle A_{\text {fast }}(q)$ and $\left\langle I_{\text {slow }}(q)\right\rangle=\langle I(q)\rangle\left[1-A_{\text {fast }}(q)\right]$. The plot of $1 /\left\langle I_{\text {fast }}(q)\right\rangle$ versus $q^{2}$ or $1 /\left\langle I_{\text {slow }}(q)\right\rangle$ versus $q^{2}$ leads to a static correlation length $\left(\xi_{\text {fast }}\right.$ or $\zeta_{\text {slow }}$ ) of each relaxation mode on the basis of eq 4 .

Optical Rotation. Optical activity $(\alpha)$ defined as $[\alpha] l C$ was measured by using a commercial polarimeter (Perkir Elmer 341), where $[\alpha]$ is the specific optical rotation of the solute, $l$ is the optical path length, and $C$ is the solution concentration. ${ }^{91} \alpha-C D$ has a distinctive optical activity. Therefore, we can use the optical rotation to precisely determine the absolute content of $\alpha-C D$ in the solution before and after the filtration with a limiting resolution of $\sim 4 \times 10^{-5} \mathrm{~g} / \mathrm{mL}$.

Sound Velocity. The sound velocity $(u)$ of each aqueous solution was measured by using a commercial instrument (USK 7D Germany). The sound frequency used was $10 \mathrm{MHz}$. By varying the propagation distance $(\Delta s)$, we measured each

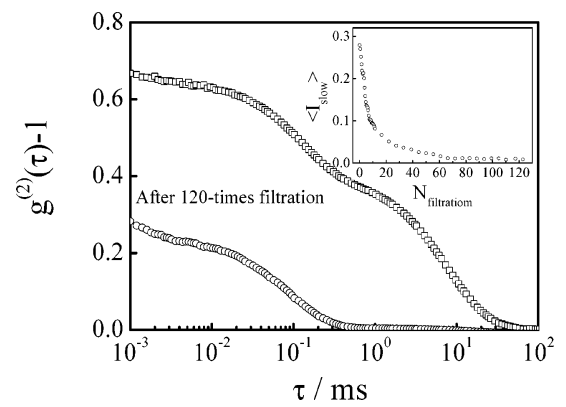

Figure 1. Normalized intensity-intensity time correlation functions $\left[g^{(2)}(\tau, q)-1\right]$ of an $\alpha$-CD aqueous solution before and after 120-times filtration. The inset shows the filtration dependence of average excess scattering intensity related to the slow mode $\left(\left\langle I(q)_{\text {slow }}\right\rangle\right)$.

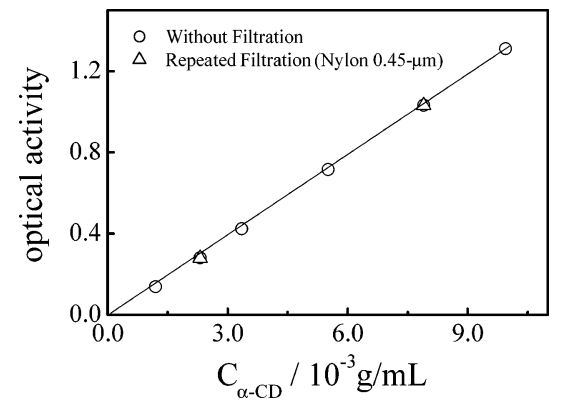

Figure 2. Concentration dependence of optical rotation of $\alpha-C D$ solutions after different treatments: $(O)$ without filtration; $(\triangle)$ after 120 -times repeated filtration $(0.45 \mu \mathrm{m}$ Nylon filter).

corresponding propagation time $(\Delta t)$ so that $u$ could be precisely determined from the slope of $\Delta s$ versus $\Delta t$ with an error less than $1 \mathrm{~m} / \mathrm{s}$. For pure water at $25^{\circ} \mathrm{C}$, our measured sound velocity is $1500 \mathrm{M} / \mathrm{s}$, very close to literature values. With a measured sound velocity, the adiabatic compressibility $\left(\kappa_{\mathrm{S}}\right)$ of a solution can be obtained using the Laplace equation: ${ }^{92}$

$$
\kappa_{\mathrm{S}}=\frac{1}{\rho u^{2}}
$$

where $\rho$ is the solution density. Therefore, we can estimate the adiabatic compressibility difference by measuring $u$ before and after the repeated filtration. Further, the isothermal compressibility $\left(\kappa_{\mathrm{T}}\right)$ can also be calculated on the basis of

$$
\kappa_{\mathrm{T}}-\kappa_{\mathrm{S}}=\frac{\alpha^{2} T}{C_{p} \rho}=\delta
$$

where $\alpha$ is the expansion factor and $C_{p}$ is the heat capacity. ${ }^{93}$

\section{Results and Discussion}

Figure 1 shows that the measured normalized intensityintensity time correlation functions of the $\alpha-C D$ aqueous solutions contain two relaxation modes. It also clearly shows that the slow mode can be completely removed after 120-times filtration by the pump in a closed circular system described before. The inset shows how the average scattering intensity $\left(\left\langle I_{\text {slow }}\right\rangle\right)$ related to the slow mode deceases as the filtration proceeds. To answer whether the repeated filtration alternates the $\alpha$-CD concentration, we measured the absolute content of $\alpha-C D$ in the solution before and after different numbers of filtrations from the optical activity $(\alpha)$ of $\alpha-C D$, as shown in Figure 2. As expected, the filtration has no effect on the $\alpha-C D$ concentration because $\alpha-\mathrm{CD}$ is a small molecule. The further 


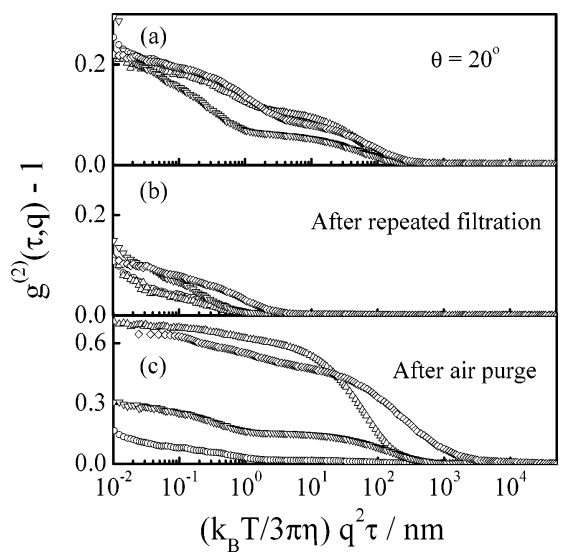

Figure 3. Normalized intensity-intensity time correlation functions $\left[g^{(2)}(\tau, q)-1\right]$ of different aqueous solutions: $(\bigcirc)$ ethanol $\left(C_{\text {ethanol }}=\right.$ $\left.1.81 \times 10^{-1} \mathrm{~g} / \mathrm{mL}\right) ;(\Delta)$ THF $\left(C_{\mathrm{THF}}=6.09 \times 10^{-2} \mathrm{~g} / \mathrm{mL}\right) ;(\nabla)$ urea $\left(C_{\text {urea }}=6.00 \times 10^{-2} \mathrm{~g} / \mathrm{mL}\right) ;(\square) \alpha-\mathrm{CD}\left(C_{\alpha-\mathrm{CD}}=2.60 \times 10^{-3} \mathrm{~g} / \mathrm{mL}\right)$. Scattering angle, $\theta=20^{\circ}$.

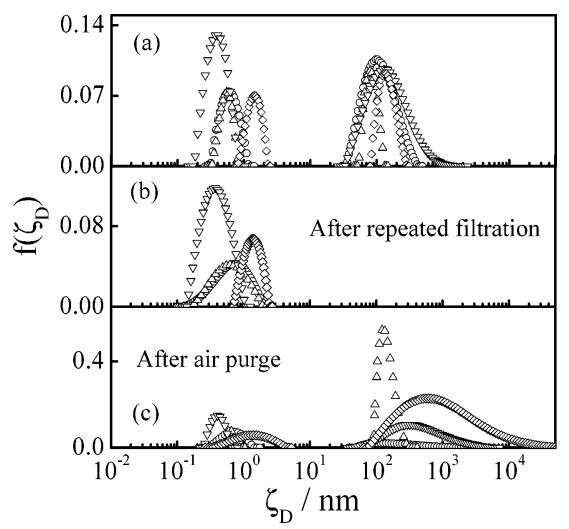

Figure 4. Normalized distributions of dynamic correlation length $\left(\xi_{\mathrm{D}}\right)$ calculated from the Laplace inversion of each corresponding $g^{(2)}(\tau, q)$ -1 in Figure 3, where the symbols are identical to those in Figure 3.

questions about the slow mode are its nature and whether it is a universal phenomenon.

Figure $3 \mathrm{a}$ shows the time correlation functions of different aqueous solutions. Note that its $x$-axis is scaled by $k_{\mathrm{B}} T q^{2} / 3 \pi \eta$ so that Figures 3 and 4 can be directly compared. The measured time correlation functions of different aqueous solutions are similar with one fast and one slow relaxation mode. Figure $4 \mathrm{a}$ shows corresponding distributions of the dynamic correlation length $\left(\xi_{\mathrm{D}}\right)$, which is normalized by the average excess scattering intensity $\left(\langle\Delta I(q)\rangle=\langle I(q)\rangle_{\text {solution }}-\langle I(q)\rangle_{\text {water }}\right)$ of each solution. In other words, the area $(A)$ under each peak is directly related to the scattering intensity from each relaxation mode. The fast mode has a dynamic correlation length $\left(\xi_{\mathrm{D} \text {,fast }}\right)$ of $0.6-1.6 \mathrm{~nm}$, attributed to a single or maybe a few associated solute molecules in water because $\zeta_{\mathrm{D}}$, fast is similar to the size of solute molecules. ${ }^{62,63}$ Such a fast relaxation mode was also observed by other experimental methods. ${ }^{94,95}$ It has been predicted and confirmed by computer simulations. ${ }^{96,97}$ On the other hand, $\zeta_{\mathrm{D} \text {,slow }}$ is in the range $100-160 \mathrm{~nm}$. There has been no conclusive explanation for the slow mode. One speculation was the complexation of a number of water molecules with some small organic molecules, that is, the formation of some kinds of supramolecular structures.

Figure $3 \mathrm{~b}$ shows that the slow mode in all solutions studied can be removed by the repeated filtration. Note that the repeated filtration has no significant effect on the fast mode, as shown in both Figures $3 \mathrm{~b}$ and $4 \mathrm{~b}$. It should be stated that the slow mode did not reappear in these solutions for a long time after

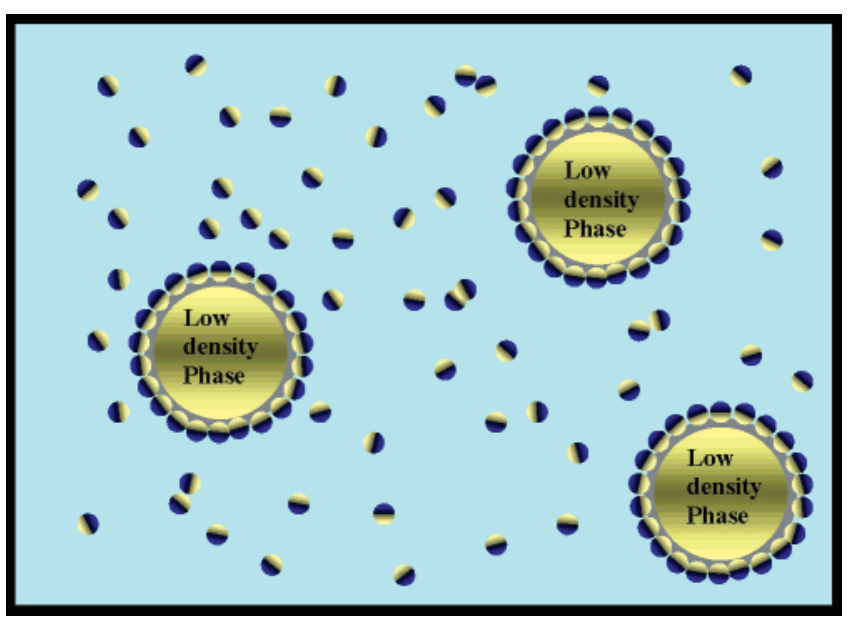

Figure 5. Schematic of nanobubbles in an aqueous solution. Small two-color spheres represent small amphiphilic organic molecules.

its removal. In other words, the solutions were very stable. There was no change in both the total scattering intensity $(\langle I(q)\rangle)$ and the intensity-intensity time correlation function even 3 days after the repeated filtration. This has not ruled out possible complexation between water and small organic molecules, since there might be an equilibrium between the complex and unimers. The next experiment was crucially important. Namely, we found that gently injecting dust-free air into each slow-mode-free solution can bring the slow relaxation mode back, as shown in both Figures $3 \mathrm{c}$ and $4 \mathrm{c}$. Note that the peaks related to these reappearing slow modes are generally larger and broader than those in Figure 4a. This is because we injected air inside.

A comparison of $f\left(\zeta_{\mathrm{D}}\right)$ in Figure 4 clearly reveals that the slow mode can be removed and regenerated, respectively, by the repeated filtration and air injection. It indicates that the slow mode is related to small bubbles, presumably stabilized by small organic molecules soluble in water. Note that the formation of these small bubbles is not affected by how these solutions were prepared. Strictly speaking, there is no surprise because these small water-soluble organic molecules are amphiphilic in nature. They can be adsorbed at the gas/water interface of each nanobubble. It is worth noting that the dissolution of a trace amount of gas in water and the formation of these nanobubbles in an aqueous solution are completely different in nature. Namely, nanobubbles can be viewed as many low density microphase domains in the solution, as schematically shown in Figure 5. Assuming that their average density is $\rho_{\mathrm{I}}$, we can rewrite the first term of eq 7 as follows

$$
k_{\mathrm{B}} T \rho \kappa_{\mathrm{T}}=k_{\mathrm{B}} T\left[f \rho_{\mathrm{I}}+(1-f) \rho_{\mathrm{II}}\right]\left[f \kappa_{\mathrm{T}, \mathrm{I}}+(1-f) \kappa_{\mathrm{T}, \mathrm{II}}\right]
$$

where the subscripts I and II denote nanobubbles and their surrounding aqueous solution, respectively, and $f$ is the volume fraction of low density nanobubbles. The second-order term of eq 18 can be omitted when $f \ll 1$. In addition, note that in general $\rho_{\mathrm{I}} \ll \rho_{\mathrm{II}}$ and $\kappa_{\mathrm{T}, \mathrm{I}} \gg \kappa_{\mathrm{T}, \mathrm{II}}$. Therefore, eq 18 can be further rewritten as

$$
\rho k_{\mathrm{B}} T \kappa_{\mathrm{T}}=k_{\mathrm{B}} T \rho_{\mathrm{II}}\left(\kappa_{\mathrm{T}, \mathrm{II}}+f \kappa_{\mathrm{T}, \mathrm{I}}\right)
$$

The second term of eq 7 can be further split into two parts as follows

$$
C\left(\frac{\partial C}{\partial \Pi}\right)_{V, T}=\frac{1}{N_{\mathrm{A}} k_{\mathrm{B}} T}\left(C_{\mathrm{f}} M+C_{\mathrm{i}}\left\langle n_{\mathrm{b}}\right\rangle M\right)
$$




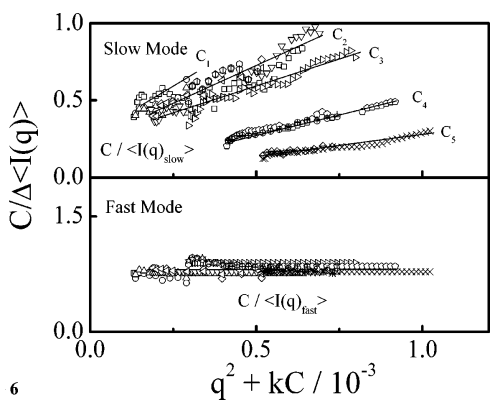

Figure 6. Scattering vector $\left(q^{2}\right)$ dependence of average excess scattering intensity related to the fast mode $\left(\left\langle I(q)_{\text {fast }}\right\rangle\right)$ and the slow mode $\left(\left\langle I(q)_{\text {slow }}\right\rangle\right)$ from different $\alpha-\mathrm{CD}$ solutions, where $C$ is the $\alpha-\mathrm{CD}$ concentration.

where $N_{\mathrm{A}}$ is Avogadro's number, $C_{\mathrm{f}}$ and $C_{\mathrm{i}}$ are the concentrations of small organic molecules free in water and at the gas/ water interface, respectively, with $C=C_{\mathrm{f}}+C_{\mathrm{i}}, M$ is the molecular weight of small organic molecules, and $\left\langle n_{\mathrm{b}}\right\rangle$ is the average number of small solute molecules at the gas/water interface of each nanobubble. $C_{\mathrm{i}}$ is related to $\left\langle n_{\mathrm{b}}\right\rangle$ and the average radius $\left(R_{\mathrm{b}}\right)$ of nanobubbles as follows

$$
C_{\mathrm{i}}=\frac{4 f\left\langle n_{\mathrm{b}}\right\rangle M}{3 \pi R_{\mathrm{b}}^{3} N_{\mathrm{A}}}
$$

A combination of eqs 7 and 18-21 leads to the excess scattering intensity $(\langle\Delta I(0)\rangle)$ at zero angle as

$$
\begin{aligned}
\langle\Delta I(0)\rangle & =\left\langle I_{\mathrm{b}}(0)\right\rangle+\left\langle I_{\mathrm{f}}(0)\right\rangle+\left\langle I_{\mathrm{i}}(0)\right\rangle \\
& =K_{1} k_{\mathrm{B}} T \rho_{\mathrm{II}} f \kappa_{\mathrm{T}, \mathrm{I}}+K_{2} C_{\mathrm{f}} M+\frac{4 K_{2} f\left\langle n_{\mathrm{b}}\right\rangle^{2} M^{2}}{3 \pi R_{\mathrm{b}}{ }^{3} N_{\mathrm{A}}}
\end{aligned}
$$

where the subscripts $\mathrm{b}, \mathrm{f}$, and i denote nanobubbles, small solute molecules free in water, and small solute molecules at the gas/ liquid interface, respectively. $\rho_{\mathrm{II}}$ is the density of the solvent (water), and $K_{1}$ and $K_{2}$ can be experimentally eliminated. On the other hand, we also have

$$
\langle\Delta I(0)\rangle=\left\langle I_{\text {fast }}(0)\right\rangle+\left\langle I_{\text {slow }}(0)\right\rangle
$$

where $\left\langle I(0)_{\text {fast }}\right\rangle$ and $\left\langle I(0)_{\text {slow }}\right\rangle$ can be calculated from a combination of static and dynamic LLS results. A comparison of eqs 22 and 23 results in

$$
\left\langle I_{\text {fast }}(0)\right\rangle=K_{2} C_{\mathrm{f}} M
$$

and

$$
\left\langle I_{\text {slow }}(0)\right\rangle=K_{1} k_{\mathrm{B}} T \rho_{\mathrm{II}} f \kappa_{\mathrm{T}, \mathrm{I}}+\frac{4 K_{2} f\left\langle n_{\mathrm{b}}\right\rangle^{2} M^{2}}{3 \pi R_{\mathrm{b}}{ }^{3} N_{\mathrm{A}}}
$$

Figure 6 shows the scattering-angle dependence of both $\left\langle I(q)_{\text {fast }}\right\rangle$ and $\left\langle I(q)_{\text {slow }}\right\rangle$. As expected, $\left\langle I(q)_{\text {fast }}\right\rangle$ is independent of the scattering angle because of its short correlation length $(<10$ $\mathrm{nm})$. Therefore, it is impossible to determine the static correlation length related to the fast relaxation mode in static LLS because $1 / q>\left\langle\zeta_{\text {D,fast }}\right\rangle$. On the other hand, each plot of $1 /\left\langle I(q)_{\text {slow }}\right\rangle$ versus $q^{2}$ leads to a static correlation length $\left(\left\langle\zeta_{\mathrm{S}}\right.\right.$,slow $\left.\rangle\right)$ related to the slow mode. Note that $\left\langle\xi_{\mathrm{S} \text {, slow }}\right\rangle$ characterizes the density correlation, a direct measurement of the average dimension of nanobubbles in these solutions. It is clear that the solutions are inhomogenous at this length scale.

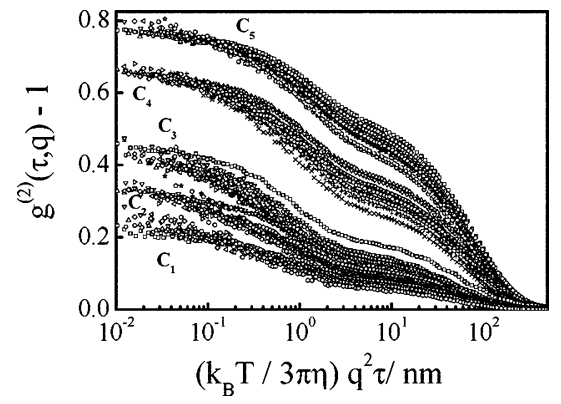

Figure 7. Concentration and scattering angular dependence of normalized intensity-intensity time correlation functions $\left[g^{(2)}(\tau, q)-1\right]$ of $\alpha$-CD solutions, where $C_{1}=2.31 \times 10^{-3} \mathrm{~g} / \mathrm{mL}, C_{2}=3.35 \times 10^{-3}$ $\mathrm{g} / \mathrm{mL}, C_{3}=5.50 \times 10^{-3} \mathrm{~g} / \mathrm{mL}, C_{4}=7.90 \times 10^{-3} \mathrm{~g} / \mathrm{mL}$, and $C_{5}=$ $9.99 \times 10^{-3} \mathrm{~g} / \mathrm{mL}$; the scattering angle $(\theta)$ ranges from 20 to $70^{\circ}$.

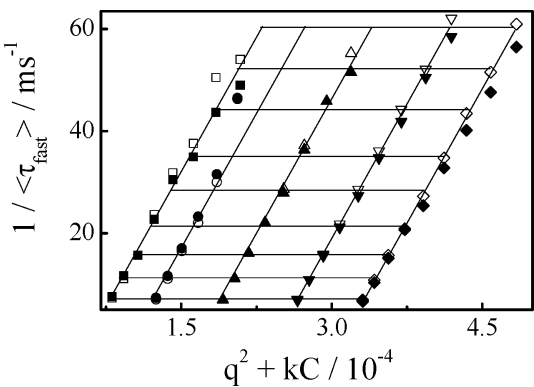

Figure 8. Scattering vector $\left(q^{2}\right)$ and polymer concentration dependence of the average characteristic decay time of the fast mode $\left(\left\langle\tau_{\text {fast }}(q)\right\rangle\right)$ calculated from two different fittings of measured $\left[g^{(2)}(\tau, q)-1\right]$ (CONTIN and double exponential).

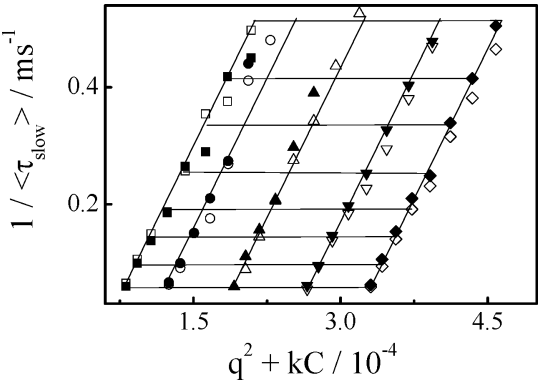

Figure 9. Scattering vector $\left(q^{2}\right)$ and polymer concentration dependence of the average characteristic decay time of the slow mode $\left(\left\langle\tau_{\text {slow }}(q)\right\rangle\right)$ calculated from two different fittings of measured $\left[g^{(2)}(\tau, q)-1\right]$ (CONTIN and double exponential).

In dynamic LLS, Figure 7 shows the concentration and angular dependence of the measured time correlation functions. Both the fast and slow modes are clear, and they have two distinct length scales. In order to minimize the fitting error, we used both the CONTIN Laplace inversion and double exponential methods to analyze each measured time correlation function. Both of them can lead to the average characteristic decay times $\left(\left\langle\tau_{\text {fast }}(q)\right\rangle\right.$ and $\left.\left\langle\tau_{\text {slow }}(q)\right\rangle\right)$, respectively, for the fast and slow modes. Figures 8 and 9 , respectively, show the dynamic Zimm plot of $1 /\left\langle\tau_{\text {fast }}(q)\right\rangle$ and $1 /\left\langle\tau_{\text {slow }}(q)\right\rangle$, which condense both the concentration and angular dependence on a single grid. Note that different fitting methods result in a similar result. The plot of $1 /\langle\tau\rangle$ versus $q^{2}$ is a straight line passing through the origin, indicating that both the fast and the slow relaxation are diffusive. The slopes, respectively, lead to the translational diffusion coefficients $\left\langle D_{\text {fast }}\right\rangle$ and $\left\langle D_{\text {slow }}\right\rangle$. Figures 8 and 9 also reveal that both $\left\langle D_{\text {fast }}\right\rangle$ and $\left\langle D_{\text {slow }}\right\rangle$ are independent of the $\alpha-C D$ concentration. Using the Stokes-Einstein equation, 


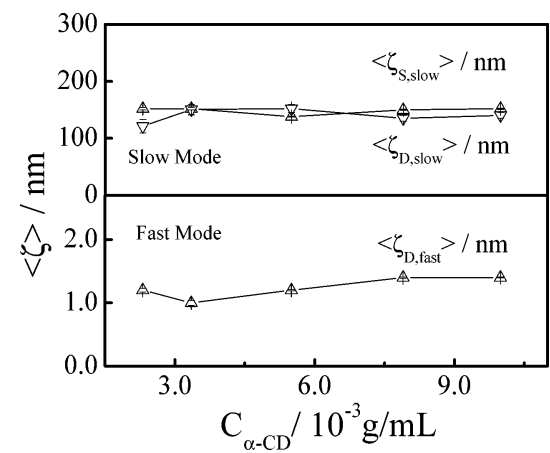

Figure 10. Concentration dependence of average static and dynamic correlation lengths $\left(\left\langle\zeta_{\text {D,fast }}\right\rangle\right)$ and $\left(\left\langle\zeta_{\text {s,slow }}\right\rangle,\left\langle\zeta_{\text {D,slow }}\right\rangle\right)$ in $\alpha-C D$ solutions. Note that $\left\langle\zeta_{\mathrm{s}, \text { fast }}\right\rangle$ is too short to be observed by LLS.

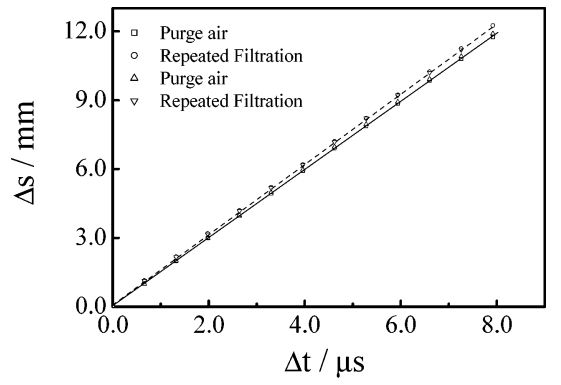

Figure 11. Plot of sound travel distance $(\Delta s)$ versus sound travel time $(\Delta t)$ in $\alpha$-CD solutions, respectively, after repeated filtration (dashed line) and after air injection (solid line).

we estimate the average dynamic correlation length of the fast mode $\left(\left\langle\zeta_{\mathrm{D} \text {,fast }}\right\rangle\right)$ as $1.2 \pm 0.1 \mathrm{~nm}$, very close to the molecular size of $\alpha$-CD. ${ }^{98}$ The constant value of $\left\langle D_{\text {slow }}\right\rangle$ indicates that the average size of nanobubbles is not affected by the $\alpha-C D$ concentration.

Figure 10 summarizes the concentration dependence of different average correlation lengths, $\left\langle\zeta_{\mathrm{D}, \text { fast }}\right\rangle,\left\langle\zeta_{\mathrm{D}, \text { slow }}\right\rangle$, and $\left\langle\zeta_{\mathrm{S} \text {,slow }}\right\rangle$. It is clear that all of them are independent of the $\alpha-C D$ concentration. It is even more important to note that the ratio of $\left\langle\zeta_{\text {S,slow }}\right\rangle /\left\langle\zeta_{\text {D,slow }}\right\rangle$ is $\sim 1$ in the whole concentration range, indicating a hollow structure. Since the slow mode (low density nanobubbles) can be completely removed by the repeated filtration, there should be a difference between the solution's adiabatic compressibilities after and before the repeated filtration. The difference $\left(\Delta \kappa_{\mathrm{S}}\right)$ should be related to the adiabatic compressibility of the low density nanobubble phase $\left(\kappa_{\mathrm{S}, \mathrm{I}}\right)$ as

$$
\frac{\Delta \kappa_{\mathrm{S}}}{\kappa_{\mathrm{T}, \mathrm{II}}}=f \frac{\kappa_{\mathrm{S}, \mathrm{I}}}{\kappa_{\mathrm{T}, \mathrm{II}}}
$$

Figure 11 shows the plot of sound travel distance $(\Delta s)$ versus the sound travel time $(\Delta t)$. The slope leads to the sound velocity in the solution. The sound velocity of each aqueous solution slightly increases after the repeated filtration but decreases after the air injection. It further confirms that the low density phase of the solution is related to nanobubbles. Our results lead to the value of $f \kappa_{\mathrm{S}, \mathrm{I}} / \kappa_{\mathrm{T}, \mathrm{II}}=8.8 \times 10^{-2}$, where $\kappa_{\mathrm{T}}$ was calculated by using eq 17 . Note that the difference between isothermal and adiabatic compressibility is independent of $f$ when $f \ll 1$ so that $\Delta \kappa_{\mathrm{S}}=\Delta \kappa_{\mathrm{T}}$ and $f \kappa_{\mathrm{S}, \mathrm{I}} / \kappa_{\mathrm{T}, \mathrm{II}}=f \kappa_{\mathrm{T}, \mathrm{I}} / \kappa_{\mathrm{T}, \mathrm{II}}$ in our experiments. A combination of eqs 24 and 25 leads to

$$
\left(\frac{\left\langle I_{\text {fast }}(0)\right\rangle}{\left\langle I_{\mathrm{w}}\right\rangle}\right) /\left(\frac{\left\langle I_{\text {slow }}(0)\right\rangle}{\left\langle I_{\mathrm{w}}\right\rangle}-\frac{f \kappa_{\mathrm{T}, \mathrm{I}}}{\kappa_{\mathrm{T}, \mathrm{II}}}\right)=\frac{3 \pi N_{\mathrm{A}} R_{\mathrm{b}}{ }^{3} C}{4 f\left\langle n_{\mathrm{b}}\right\rangle^{2} M}
$$

where $\left\langle I_{\mathrm{w}}\right\rangle$ is the scattering intensity from pure water and $C$ is the solution concentration. Note that we have dropped $1 /\left\langle n_{\mathrm{b}}\right\rangle$ because $\left\langle n_{\mathrm{b}}\right\rangle \gg 1$. Each term on the left side of eq 27 and $R_{\mathrm{b}}$ can be measured. Therefore, we estimated $f\left\langle n_{\mathrm{b}}\right\rangle^{2} \sim 1.7 \times 10^{4}$ for the $\alpha$-CD aqueous solution $\left(C=7.90 \times 10^{-3} \mathrm{~g} / \mathrm{mL}\right)$. In these experimentally determined $f \kappa_{\mathrm{T}, \mathrm{I}} / \kappa_{\mathrm{T}, \mathrm{II}}$ and $f\left\langle n_{\mathrm{b}}\right\rangle^{2}$, we still have three unknown parameters $\left(f, \kappa_{\mathrm{T}, \mathrm{I}}\right.$, and $\left.\left\langle n_{\mathrm{b}}\right\rangle\right)$. It is impossible to find each of them without other additional information, but this did not prevent us from estimating their ranges. First, we estimated the maximum value of $\left\langle n_{\mathrm{b}}\right\rangle$ to be $\sim 5 \times 10^{4}$ from the average surface area per nanobubble and the size of one $\alpha-C D$ molecule by assuming only one layer of closely packed $\alpha-C D$ molecules at the gas/water interface. Using such an estimated maximum $\left\langle n_{\mathrm{b}}\right\rangle$, we have $f \geq 7.7 \times 10^{-6}$ and $\kappa_{\mathrm{T}, \mathrm{I}} / \kappa_{\mathrm{T}, \mathrm{II}} \leq 1.2 \times$ $10^{5}$. We can further estimate the internal pressure $(P)$ to be higher than 2 atm from $\kappa_{\mathrm{T}, \mathrm{I}} / \kappa_{\mathrm{T}, \mathrm{II}}$ by assuming $\kappa_{\mathrm{T}, \mathrm{I}}=1 / P$ (ideal gas).

On the other hand, the maximum averaged internal pressure $\left(P_{\max }\right)$ inside each nanobubble can be estimated by using the Laplace-Young equation:

$$
\Delta P=\frac{2 \gamma}{R}
$$

where $\Delta P$ is the pressure difference between the inside and outside of nanobubbles, $\gamma$ is the surface tension of water, and $R$ is the average radius of nanobubbles. As expected, small amphiphilic solute molecules at the gas/water interface reduce $\gamma$. Therefore, $f_{\max }=7.6 \times 10^{-5}, P_{\max }=19 \mathrm{~atm}$, and $\left\langle n_{\mathrm{b}}\right\rangle>1.5$ $\times 10^{4}$. Note that the coverage of small amphiphilic organic molecules at the gas/water interface greatly reduces the pressure inside nanobubbles. This might explain why they can form and also become rather stable in small organic molecule aqueous solutions.

\section{Conclusions}

By using a combination of LLS and isothermal compressibility measurements, we found that, in small water-soluble organic molecule aqueous solutions, the slow mode observed in dynamic LLS is attributed to the spontaneous formation of small bubbles $(\sim 100 \mathrm{~nm})$, not as previously suggested, due to their complexation with water to form large water/solute supramolecular structures. These nanobubbles can be viewed as low density microdomains in the solution, stabilized by a layer of small organic molecules (amphiphilic in nature) at the gas/water interface. A combination of static and dynamic LLS results enables us to estimate that the volume faction of these nanobubbles is fairly small and the internal pressure could be as low as 2 atm because the surface tension is greatly reduced by the adsorbed layer of amphiphilic solute molecules. This explains why these nanobubles are rather stable.

Acknowledgment. The financial support of the Hong Kong Special Administration Region Earmarked Grants (CUHK4025/ 04P, 2160242), the Chinese Academy of Sciences Special Grant (KJCX2-SW-H14), and the Natural National Science Foundation Project (20574065) is gratefully acknowledged.

\section{References and Notes}

(1) Stillinger, F. H. Science 1980, 209, 451

(2) Rontgen, W. C. Ann. Phys. u. Chem. 1892, 45, 91. 21,315

(3) Lang, E. W.; Ludemann, H. D. Angew. Chem., Int. Ed. Engl. 1982,

(4) Speedy, R. J.; Mezei, M. J. Phys. Chem. 1985, 89, 171.

(5) Westhof, E. Water and Biomolecular Macromolecules; CRC: Boca Raton, FL, 1993. 
(6) Franks, F. Water: a matrix of life, 2nd ed.; Royal Society of Chemistry: Cambridge, U.K., 2000.

(7) Karnicky, J. F.; Pings, C. J. In Advances in Chemical Physics; Prigogine, I., Rice, S.A., Eds.; Wiley: New York, 1976; Vol. 34.

(8) G. Hura, J. M.; Sorenson, R. M.; Glaeser, T. H. G. J. Chem. Phys. 2000, 113, 9140.

(9) Sorenson, J. M.; Hura, G.; Glaeser, R. M. J. Chem. Phys. 2000, 113,9149 . 5818 .

(10) Bassez, M. P.; Lee, J.; Robinson, G. W. J. Phys. Chem. 1987, 91,

(11) Robinson, G. W. Water in biology, chemistry, and physics: experiment overviews and computational methodologies; World Scientific: Singapore, 1996.

(12) Schweler, E.; Galli, F. Phys. Rev. Lett. 2000, 84, 2429.

(13) Silvestrelli, P. L.; Parrinello, M. J. Chem. Phys. 1999, 111, 3572

(14) Sprik, M.; Hutter, J.; Parrinello, M. J. Chem. Phys. 1996, 105, 1142.

(15) Jorgensen,W. L.; Chandrasekhar, J.; Madura, J. D.; Impey, R. W.; Klein, M. L. J. Chem. Phys. 1983, 79, 926.

(16) Cho, C. H.; Singh, S.; Robinson, G. W. The Royal Society of Chemistry Faraday Division General Discussion No. 103; Sheffield: England, 1996.

(17) Cho, C. H.; Singh, S.; Robinson, G. W. Phys. Rev. Lett. 1996, 76 1651.

(18) Cho, C. H.; Singh, S.; Robinson, G. W. J. Chem. Phys. 1997, 107, 7979.

(19) Kamb, B. Structural Chemistry and Molecular Biology; W. H. Freeman: San Franciso, CA, 1968.

(20) Head, G.; Hura, G. Chem. Rev. 2002, 102, 2651. (21)

(21) Franks, F. Water, a comprehensive treatise; Plenum: New York,

(22) Bellissent-Funel, M.C.; Dore, J. C. Hydrogen bond networks; Kluwer Academic: Boston, MA, 1991.

(23) Widom, B.; Bhimalapuram, P.; Koga, K. Phys. Chem. Chem. Phys. 2003, 5,3085

(24) Smith, D. E.; Haymet, A. D. J. J. Chem. Phys. 1993, 98, 6445

(25) Guillot, B.; Guissani, Y. J. Chem. Phys. 1993, 99, 8075.

(26) Besseling, N. A. M.; Lyklema, J. J. Phys. Chem. B 1997, 101, 7604

(27) Silverstein, K. A. T.; Haymet, A. D. J.; Dill, K. A. J. Chem. Phys. 1999, 111,8000 .

(28) Pratt, L. R. Encyclopedia of Computational Chemistry: "Hydrophobic Effects"; Wiley: Chichester, U.K., 1998.

(29) Chandler, D. Phys. Rev. E 1993, 48, 2898.

(30) Tanford, C. The Hydrophobic Effect: Formation of Micelles and Biological Membranes; Wiley: New York, 1980.

(31) Kauzmann, W. Adv. Protein Chem. 1959, 14, 1

(32) Dill, K. A. Biochemistry 1990, 29, 7133.

(33) Blokzijl, W.; Engberts, J. B. F. N. Angew. Chem., Int. Ed. Engl. 1993, 32, 1545 .

(34) Fink, A. L. Folding Des. 1998, 3, R9.

(35) Pratt, L. R. Annu. Rev. Phys. Chem. 2002, 53, 409

(36) Hummer, G.; Garde, S.; Garcia, A. E.; Pohorille, A.; Pratt, L. R. Proc. Natl. Acad. Sci. U.S.A. 1996, 93, 8951.

(37) Garde, S.; Hummer, A. E.; Paulaitis, M. E.; Pratt, L. R. Phys. Rev. Lett. 1996, 77, 4966.

(38) Hummer, G.; Garde, S.; Garcia, A.; Pratt, L. R. Chem. Phys. 2000, $258,349$.

(39) Shore, J. E.; Johnson, R. W. IEEE Trans. Inf. Theory 1980, IT-26, 26.

(40) Jaynes, E. T. In Papers on Probability, Statistics and Statistical Physics; Rosenkrantz, R. D., Ed.; 315.

(41) Lum, K.; Chandler, D.; Weeks, J. D. J. Phys. Chem. B 1999, 103 4570 .

(42) Choudhury, N.; Pettitt, B. M. J. Am. Chem. Soc. 2005, 127, 3556.

(43) Moghaddam, M. S.; Shimizu, S.; Chan, H. C. J. Am. Chem. Soc. 2005, 127, 303

(44) Ghosh, T.; Garcia, A. E.; Garde, S. J. Phys. Chem. B 2003, 107 612.

(45) Czaplewski, C.; Liwo, A.; Ripoll, D. R.; Scheraga, H. A. J. Phys. Chem. B 2005, 109, 8108 .

(46) Hummer, G.; Shekhar, G. Phys. Rev. Lett. 1998, 80, 4193.

(47) Vossen, M.; Forstmann, F. J. Chem. Phys. 1994, 101, 2379.

(48) Wallqvist, A.; Gallicchio, E.; Levy, R. M. J. Phys. Chem. B 2001, 105,6745 .

(49) Jan van Oss, C. J. Mol. Recognit 2003, 16, 177

(50) Wood, J.; Sharma, R. Langmuir 1995, 11, 4797.
(51) Sorensen, C. M. J. Phys. Chem. 1988, 92, 2367.

(52) Oleinikova, A.; Weingartner, H. Phys. Chem. Chem. Phys. 2002, $4,955$.

(53) Murthy, S. S. N. J. Phys. Chem. A 1999, 103, 7927.

(54) Marbach, W.; Asaad, A. N.; Krebs, P. J. Phys. Chem. A 1999, 103, 28.

(55) Iwasaki, K.; Fujiyama, T. J. Phys. Chem. 1979, 83, 463.

(56) Atkinson, G.; Rajagopalan, S.; Atkinson, B. L. J. Phys. Chem. 1981, 85,733

(57) Takamuku, T.; Nakamizao, A.; Tabata, M.; Yoshida, K.; Yamaguchi T.; Otomo, T. J. Mol. Liq. 2003, 103-104, 143.

(58) Fukasawa, T. J. Chem. Phys. 2003, 118, 6387.

(59) Nose, A. J. Phys. Chem. B 2004, 108, 798.

(60) Idrissi, A. Spectrochim. Acta, Part A 2005, 61, 1.

(61) Idrissi, A.; Sokolic, F.; Perera, A. J. Chem. Phys. 2000, 112, 9479

(62) Sedlak, M. J. Phys. Chem. B 2006, 110, 4329.

(63) Gonzalez-Gaitano, G.; Rodriguez, P.; Isasi, J. R.; Fuentes, M.; Tardajos, G.; Sanchez, M. J. Inclusion Phenom. Macrocyclic Chem. 2002 $44,101$.

(64) Euliss, G. W.; Sorensen, C. M. J. Chem. Phys. 1984, 80, 4767 468 .

(66) Meagher, L.; Craig, V. S. J. Langmuir 1994, 10, 2736

(67) Attard, P. Langmuir 1996, 12, 1693.

(68) Eriksson, J. C.; Ljunggren, S.; Claesson, P. M. J. Chem. Soc., Faraday Trans. 2 1989, 85, 163.

(69) Podgornik, R. J. Phys. Chem. 1989, 92, 5840

(70) Tyrrell, J. W. G.; Attard, P. Phys. Rev. Lett. 2001, 87, 17.

(71) Stevens, H.; Considine, R. F.; Drummond, C. J.; Hayes, R. A.; Attard, P. Langmuir 2005, 21, 6399.

(72) Christenson, H. K.; Claesson, P. M. Adv. Colloid Interface Sci. 2001, 91, 391.

(73) Bilgram, J. H.; Güttinger, H.; Känzig, W. Phys. Rev. Lett. 1978, $40,1394$.

(74) Willimas, L. M.; Srinivasan, M. R.; Cummins, H. Z. Phys. Rev. Lett. 1990, 64, 1526.

(75) Henderson, D. Fundamentals of Inhomogeneous Fluids; M. Dekker: New York, 1992.

(76) Lugli, F.; Hofinger, S.; Zerbetto, F. J. Am. Chem. Soc. 2005, 127, 8020

(77) Lum, K.; Chandler, D.; Weeks, J. D. J. Phys. Chem. B 1999, 103, 4750.

(78) Ljunggren, S.; Reiksson, J. C. Colloids Surf., A 1997, 151, 129.

(79) Levy, M. J. Am. Chem. Soc. 1962, 84, 1345.

(80) Chu, B. Laser Light Scattering, 2nd ed.; Academic: New York, 1991.

(81) DuBois, S. Photon Correlation Techniques in Fluid Mechanics; Springer-Verlag: Berlin, 1983.

(82) Brown, W. Light Scattering; Clarendon: Oxford, U.K., 1996.

(83) Plischke, M.; Bergersen, B. In Equlibrium Statistical Physics, 2nd ed.; World Scientific: 1994.

(84) Reichl, L. E. A Modern Course in Statistical Physics; Edward Arnold: 1980.

(85) Berne, B.; Pecora, R. Dynamic Light Scattering; Plenum: New York, 1976.

(86) Sikharulidze, I.; Jeude, W. H. Phys. Rev. E 2005, 72, 011704-1.

(87) Cantrell, C. D. Phys. Rev. A 1970, 1, 672

(88) Siegert, A. J. F. MIT Radiation Lab. Report No. 465, 1943.

(89) Mandel, L.; Wolf, E. Rev. Mod. Phys. 1965, 37, 231.

(90) Teraoka, I. Polymer Solutions; Wiley-Interscience: New York, 1998.

(91) Daniels, F.; Mathews, J. H.; Williams, J. W.; Bender P.; Alberty,

R. A. Experiment Physical Chemistry, 5th ed.; McGraw-Hill: 1956.

(92) Diaz, D. G.; Navaza, J. M. J. Chem. Eng. Data 2006, 51, 722

(93) Kolhapurkar, R. R.; Dagade, D. H.; Pawar, R. B.; Patil, K. J. J. Chem. Thermodyn. 2006, 38, 105.

(94) Fukasawa, T.; Amo, Y.; Tominaga, Y. J. Chem. Phys. 2003, 118, 6387.

(95) Fukasawa, T.; Tominaga, Y.; Wakisaka, A. J. Phys. Chem. A 2004, 108,59 .

(96) Fretias, L. C. G.; Corderio, J. M. M. J. Mol. Struct. 1995, 335, 189.

(97) Sahu, A. P. K.; Chaud, H.; Lee, S. L. Chem. Phys. Lett. 2004, 386, 351.

(98) Szejtli, J. Chem. Rev. 1998, 98, 1743. 